\section{S sciendo}

DOI: $10.2478 /$ awutm-2018-0020
Analele Universităţii de Vest,

Timişoara

Seria Matematică - Informatică

LVI, 2, (2018), 131- 150

\title{
On $\Psi$ Bounded Solutions for a Nonlinear Lyapunov Matrix Differential Equation on $\mathbb{R}$
}

Aurel Diamandescu

Dedicated to Professor Mihail Megan on the occasion of his 70th birthday

\begin{abstract}
Using Banach and Schauder - Tychonoff fixed point theorems, existence results for a nonlinear Lyapunov matrix differential equation on $\mathbb{R}$ are given. The obtained results generalize and extend the results from [5] and [18].
\end{abstract}

AMS Subject Classification (2000). 34C11, 34D05, 34D10.

Keywords. $\Psi$ - boundedness on $\mathbb{R}$, nonlinear Lyapunov matrix differential equation on $\mathbb{R}$.

\section{Introduction}

The purpose of present paper is to provide sufficient conditions for the existence and uniqueness and existence of at least one $\Psi$ - bounded solution for the nonlinear Lyapunov matrix differential equation on $\mathbb{R}$

$$
Z^{\prime}=A(t) Z+Z B(t)+C(t)+F(t, Z)
$$

with the help of Banach and Schauder-Tychonoff fixed point theorems.

We first establish two results in connection with the existence and uniqueness and existence of at least one $\Psi$ - bounded solution for the nonlinear matrix differential equation on $\mathbb{R}$ of the form

$$
Z^{\prime}=A(t) Z+C(t)+F(t, Z)
$$


Second, using vectorization operator and Kronecker product of matrices, we treat the same problems for the nonlinear Lyapunov matrix differential equation on $\mathbb{R}$ of the form (1.1).

History of problem. A classical result in connection with boundedness of solutions of systems of ordinary differential equations

$$
x^{\prime}=A(t) x+c(t)+f(t, x)
$$

was given by Coppel [5] (Chapter V, section 2, Theorem 4). The problem of $\Psi$ - bounded solutions for systems of ordinary differential equations has been studied by many authors: [2], [3], [4], [8], [9], [12], [13], [14], [18] and for Lyapunov matrix differential equations, [6], [7], [10], [11], [16], [17].

The introduction of the matrix function $\Psi$ in the study of solutions permits to obtain a mixed asymptotic behavior of the components of the solutions of the above equations.

\section{Preliminaries}

In this section we present some basic notations, definitions, hypotheses and results which are useful later on.

Let $\mathbb{R}^{d}$ be the Euclidean $d$ - dimensional space. For $x=\left(x_{1}, x_{2}, \ldots, x_{d}\right)^{T} \in$ $\mathbb{R}^{d}$, let $\|x\|=\max \left\{\left|x_{1}\right|,\left|x_{2}\right|, \ldots,\left|x_{d}\right|\right\}$ be the norm of $x$ (here, ${ }^{T}$ denotes transpose).

Let $\mathbb{M}_{d \times d}$ be the linear space of all real $d \times d$ matrices.

For $A=\left(a_{i j}\right) \in \mathbb{M}_{d \times d}$, we define the norm $|A|$ by $|A|=\sup _{\|x\| \leq 1}\|A x\|$. It is well-known that $|A|=\max _{1 \leq i \leq d}\left\{\sum_{j=1}^{d}\left|a_{i j}\right|\right\}$.

By a solution of the equation (1.1) we mean a continuous differentiable $d \times d$ matrix function satisfying the equation (1.1) for all $t \in \mathbb{R}$.

In equation (1.1) we assume that the coefficients are continuous functions.

Let $\Psi_{i}: \mathbb{R}_{+} \longrightarrow(0, \infty), i=1,2, \ldots, d$, be continuous functions and

$$
\Psi=\operatorname{diag}\left[\Psi_{1}, \Psi_{2}, \cdots \Psi_{d}\right] .
$$

A matrix $P$ is said to be a projection if $P^{2}=P$.

Definition 2.1. ([12], [8]) A function $\varphi: R \longrightarrow R^{d}$ is said to be $\Psi$ - bounded on $R$ if $\Psi(t) \varphi(t)$ is bounded on $R$ (i.e. there exists $m>0$ such that $\|$ $\Psi(t) \varphi(t) \| \leq m$, for all $t \in R)$.

Otherwise, is said that the function $\varphi$ is $\Psi$ - unbounded on $R$. 
Definition 2.2. ([10]) A matrix function $M: \mathbb{R} \longrightarrow \mathbb{M}_{d \times d}$ is said to be $\Psi-$ bounded on $R$ if the matrix function $\Psi(t) M(t)$ is bounded on $R$ (i.e. there exists $m>0$ such that $|\Psi(t) M(t)| \leq m$, for all $t \in R$ ).

Otherwise, is said that the matrix function $M$ is $\Psi$ - unbounded on $R$.

We now describe a few definitions and properties in connection with Kronecker product of matrices and vectorization operator.

Definition 2.3. ([1]) Let $A=\left(a_{i j}\right) \in M_{m \times n}$ and $B=\left(b_{i j}\right) \in M_{p \times q}$. The Kronecker product of $A$ and $B$, written $A \otimes B$, is defined to be the partitioned matrix

$$
A \otimes B=\left(\begin{array}{cccc}
a_{11} B & a_{12} B & \cdots & a_{1 n} B \\
a_{21} B & a_{22} B & \cdots & a_{2 n} B \\
\vdots & \vdots & \vdots & \vdots \\
a_{m 1} B & a_{m 2} B & \cdots & a_{m n} B
\end{array}\right)
$$

Obviously, $A \otimes B \in \mathbb{M}_{m p \times n q}$.

We next show the important rules of calculation of the Kronecker product.

Lemma 2.1. ([1]) The Kronecker product has the following properties and rules, provided that the dimension of the matrices are such that the various expressions exist:

1). $A \otimes(B \otimes C)=(A \otimes B) \otimes C ;$

2). $(A \otimes B)^{T}=A^{T} \otimes B^{T}$

3). $(A \otimes B) \cdot(C \otimes D)=(A \cdot C) \otimes(B \cdot D)$;

4). $(A \otimes B)^{-1}=A^{-1} \otimes B^{-1}$

5). $A \otimes(B+C)=A \otimes B+A \otimes C$;

6). $(A+B) \otimes C=A \otimes C+B \otimes C$;

7). $I_{d} \otimes A=\left(\begin{array}{cccc}A & O & \cdots & O \\ O & A & \cdots & O \\ \vdots & \vdots & \vdots & \vdots \\ O & O & \cdots & A\end{array}\right)$;

8). $(A(t) \otimes B(t))^{\prime}=A^{\prime}(t) \otimes B(t)+A(t) \otimes B^{\prime}(t)$; (' denotes the derivative $\left.\frac{d}{d t}\right)$.

Proof. See in [1].

Definition 2.4. ([15]) The application Vec: $\mathbb{M}_{m \times n} \longrightarrow \mathbb{R}^{m n}$, defined by

$$
\operatorname{Vec}(A)=\left(a_{11}, a_{21}, \cdots, a_{m 1}, a_{12}, a_{22}, \cdots, a_{m 2}, \cdots, a_{1 n}, a_{2 n}, \cdots, a_{m n}\right)^{T},
$$

where $A=\left(a_{i j}\right) \in \mathbb{M}_{m \times n}$, is called the vectorization operator. 
Lemma 2.2. ([10]) The vectorization operator

$$
\text { Vec }: \mathbb{M}_{m \times n} \longrightarrow \mathbb{R}^{m n}, A \longrightarrow \operatorname{Vec}(A),
$$

is a linear and one-to-one operator. In addition, $\mathcal{V e c}$ and $\mathcal{V e c}^{-1}$ are continuous operators.

Proof. See Lemma 2, [10].

Remark 2.1. Obviously, a function $F: \mathbb{R} \longrightarrow \mathbb{M}_{d \times d}$ is a continuous (differentiable) matrix function on $R$ if and only if the function $f: R \longrightarrow \mathbb{R}^{d^{2}}$, defined by $f(t)=\operatorname{Vec}(F(t))$, is a continuous (differentiable) vector function on $R$.

We recall that the vectorization operator $V e c$ has the following properties as concerns the calculations.

Lemma 2.3. ([15]) If $A, B, M \in \mathbb{M}_{n \times n}$, then

1). $\operatorname{Vec}(A M B)=\left(B^{T} \otimes A\right) \cdot \operatorname{Vec}(M)$

2). $\operatorname{Vec}(M B)=\left(B^{T} \otimes I_{n}\right) \cdot \operatorname{Vec}(M)$;

3). $\operatorname{Vec}(A M)=\left(I_{n} \otimes A\right) \cdot \operatorname{Vec}(M)$

4). $\operatorname{Vec}(A M)=\left(M^{T} \otimes A\right) \cdot \operatorname{Vec}\left(I_{n}\right)$.

Proof. See [15], Chapter 2.

The following lemmas play a vital role in the proofs of main results of present paper.

Lemma 2.4. ([10]) The matrix function $Z(t)$ is a solution on $R$ of (1.1) if and only if the vector function $z(t)=\operatorname{Vec}(Z(t))$ is a solution of the differential system

$$
z^{\prime}=\left(I_{d} \otimes A(t)+B^{T}(t) \otimes I_{d}\right) z+c(t)+f(t, z),
$$

where $c(t)=\operatorname{Vec}(C(t))$ and $f(t, z)=\operatorname{Vec}(F(t, Z))$, on the same interval $R$.

Proof. See Lemma 7, [10].

Definition 2.5. ([10]) The above system (2.1) is called "corresponding Kronecker product system associated with (1.1)".

Lemma 2.5. ([10]). For every matrix function $M: R \longrightarrow \mathbb{M}_{d \times d}$,

$$
\frac{1}{d}|\Psi(t) M(t)| \leq\left\|\left(I_{d} \otimes \Psi(t)\right) \operatorname{Vec}(M(t))\right\|_{\mathbb{R}^{d^{2}}} \leq|\Psi(t) M(t)|, \forall t \geq 0 .
$$


Proof. See Lemma 4, [10].

Lemma 2.6. The solutions of (1.1) are $\Psi$ - bounded on $R$ if and only if the solutions of the differential system (2.1) are $I_{d} \otimes \Psi-$ bounded on $R$.

Proof. It results from above Lemma (2.5).

Lemma 2.7. ([10]). Let $X(t)$ and $Y(t)$ be a fundamental matrices for the equations

$$
\begin{aligned}
& Z^{\prime}=A(t) Z \\
& Z^{\prime}=Z B(t)
\end{aligned}
$$

respectively.

Then, the matrix $Z(t)=Y^{T}(t) \otimes X(t)$ is a fundamental matrix for the linear differential system

$$
z^{\prime}=\left(I_{d} \otimes A(t)+B^{T}(t) \otimes I_{d}\right) z
$$

(i.e. for homogeneous differential system associated with (2.1).

Proof. See Lemma 6, [10].

\section{$3 \quad \Psi$ - bounded solutions for the matrix differential equa- tion (1.2)}

The purpose of this section is to provide sufficient conditions for the existence and uniqueness and existence of at least one $\Psi$ - bounded solution on $\mathbb{R}$ for the equation (1.2).

Theorem 3.1. Suppose that:

1). There exist supplementary projections $P_{-}, P_{0}, P_{+} \in \mathbb{M}_{d \times d}$ and a positive constant $K$ such that the fundamental matrix $X(t)$ for (2.3) satisfies the condition

$$
\begin{aligned}
& \int_{-\infty}^{t}\left|\Psi(t) X(t) P_{-} X^{-1}(s) \Psi^{-1}(s)\right| d s+ \\
& +\left|\int_{0}^{t}\right| \Psi(t) X(t) P_{0} X^{-1}(s) \Psi^{-1}(s)|d s|+ \\
& +\int_{t}^{\infty}\left|\Psi(t) X(t) P_{+} X^{-1}(s) \Psi^{-1}(s)\right| d s \leq K,
\end{aligned}
$$

for all $t \geq 0$;

2). The continuous function $F: R \times \mathbb{M}_{d \times d} \rightarrow \mathbb{M}_{d \times d}$ satisfies $F(t, O)=O$ and the Lypschitz condition

$$
\left|\Psi(t)\left(F\left(t, Z_{1}\right)-F\left(t, Z_{2}\right)\right)\right| \leq \gamma\left|\Psi(t)\left(Z_{1}-Z_{2}\right)\right|,
$$


for $t \in R, Z_{1}, Z_{2} \in \mathbb{M}_{d \times d}$ with $\left|\Psi(t) Z_{1}\right| \leq \rho,\left|\Psi(t) Z_{2}\right| \leq \rho$ for $t \in R, \quad(\rho>0$ is given), where $\gamma$ is a positive constant such that $\gamma K<1$;

3). The continuous function $C: R \rightarrow \mathbb{M}_{d \times d}$ is $\Psi$ - bounded on $R$ such that

$$
|C|_{\Psi}=\sup _{t \in R}|\Psi(t) C(t)| \leq \frac{\rho(1-\gamma K)}{K} .
$$

Then, the equation (1.2) has a unique $\Psi$ - bounded solution $Z(t)$ on $R$ for which $|\Psi(t) Z(t)| \leq \rho$, for all $t \in R$.

Proof. We prove this theorem by means of Banach fixed point theorem.

Consider the space

$$
C_{\Psi}=\left\{Z: R \rightarrow \mathbb{M}_{d \times d} \mid Z \text { is continuous and } \Psi-\text { bounded on } R\right\} .
$$

$C_{\Psi}$ is a Banach space with respect to the norm $|Z|_{\Psi}=\sup _{t \in R}|\Psi(t) Z(t)|$.

Let the ball $S_{\rho}=\left\{Z \in C_{\Psi} \|\left. Z\right|_{\Psi} \leq \rho\right\}$.

For $Z \in C_{\Psi}$, define the operator $\mathrm{T}$ by

$$
\begin{aligned}
& (T Z)(t)=\int_{-\infty}^{t} X(t) P_{-} X^{-1}(s)(C(s)+F(s, Z(s))) d s+ \\
& +\int_{0}^{t} X(t) P_{0} X^{-1}(s)(C(s)+F(s, Z(s))) d s- \\
& -\int_{t}^{\infty} X(t) P_{+} X^{-1}(s)(C(s)+F(s, Z(s))) d s
\end{aligned}
$$

From hypotheses, $T Z$ exists and is continuous differentiable on $\mathrm{R}$.

For $Z \in S_{\rho}$ and $t \in R$, we have

$$
\begin{aligned}
& |\Psi(t)(T Z)(t)|=\mid \int_{-\infty}^{t} \Psi(t) X(t) P_{-} X^{-1}(s) \Psi^{-1}(s) \Psi(s)(C(s)+F(s, Z(s))) d s+ \\
& +\int_{0}^{t} \Psi(t) X(t) P_{0} X^{-1}(s) \Psi^{-1}(s) \Psi(s)(C(s)+F(s, Z(s))) d s- \\
& -\int_{t}^{\infty} \Psi(t) X(t) P_{+} X^{-1}(s) \Psi^{-1}(s) \Psi(s)(C(s)+F(s, Z(s))) d s \mid \leq \\
& \leq \int_{-\infty}^{t}\left|\Psi(t) X(t) P_{-} X^{-1}(s) \Psi^{-1}(s) \| \Psi(s)(C(s)+F(s, Z(s)))\right| d s+ \\
& +\left|\int_{0}^{t}\right| \Psi(t) X(t) P_{0} X^{-1}(s) \Psi^{-1}(s) \| \Psi(s)(C(s)+F(s, Z(s)))|d s|+ \\
& +\int_{t}^{\infty}\left|\Psi(t) X(t) P_{+} X^{-1}(s) \Psi^{-1}(s) \| \Psi(s)(C(s)+F(s, Z(s)))\right| d s \leq \\
& \leq K \cdot \sup _{t \in R}|\Psi(s)(C(s)+F(s, Z(s)))| \leq \\
& \leq K \cdot\left(\frac{\rho(1-\gamma K)}{K}+\gamma|Z| \Psi\right)=\rho(1-\gamma K)+\gamma K \rho=\rho .
\end{aligned}
$$


It follows that $T Z \in S_{\rho}$ and hence,

$$
T S_{\rho} \subset S_{\rho} .
$$

On the other hand, for $Z_{1}, Z_{2} \in S_{\rho}$ and $t \in R$, we have $\left|\Psi(t)\left(\left(T Z_{1}\right)(t)-\left(T Z_{2}\right)(t)\right)\right|=$

$=\mid\left[\int_{-\infty}^{t} \Psi(t) X(t) P_{-} X^{-1}(s) \Psi^{-1}(s) \Psi(s)\left(C(s)+F\left(s, Z_{1}(s)\right)\right) d s+\right.$

$+\int_{0}^{t} \Psi(t) X(t) P_{0} X^{-1}(s) \Psi^{-1}(s) \Psi(s)\left(C(s)+F\left(s, Z_{1}(s)\right)\right) d s-$

$\left.-\int_{t}^{\infty} \Psi(t) X(t) P_{+} X^{-1}(s) \Psi^{-1}(s) \Psi(s)\left(C(s)+F\left(s, Z_{1}(s)\right)\right) d s\right]-$

$-\left[\int_{-\infty}^{t} \Psi(t) X(t) P_{-} X^{-1}(s) \Psi^{-1}(s) \Psi(s)\left(C(s)+F\left(s, Z_{2}(s)\right)\right) d s+\right.$

$+\int_{0}^{t} \Psi(t) X(t) P_{0} X^{-1}(s) \Psi^{-1}(s) \Psi(s)\left(C(s)+F\left(s, Z_{2}(s)\right)\right) d s-$

$\left.-\int_{t}^{\infty} \Psi(t) X(t) P_{+} X^{-1}(s) \Psi^{-1}(s) \Psi(s)\left(C(s)+F\left(s, Z_{2}(s)\right)\right) d s\right] \mid=$

$=\mid \int_{-\infty}^{t} \Psi(t) X(t) P_{-} X^{-1}(s) \Psi^{-1}(s) \Psi(s)\left(F\left(s, Z_{1}(s)\right)-F\left(s, Z_{2}(s)\right)\right) d s+$

$+\int_{0}^{t} \Psi(t) X(t) P_{0} X^{-1}(s) \Psi^{-1}(s) \Psi(s)\left(F\left(s, Z_{1}(s)\right)-F\left(s, Z_{2}(s)\right)\right) d s-$

$-\int_{t}^{\infty} \Psi(t) X(t) P_{+} X^{-1}(s) \Psi^{-1}(s) \Psi(s)\left(F\left(s, Z_{1}(s)\right)-F\left(s, Z_{2}(s)\right)\right) d s \mid \leq$

$\leq \int_{-\infty}^{t}\left|\Psi(t) X(t) P_{-} X^{-1}(s) \Psi^{-1}(s) \| \Psi(s)\left(F\left(s, Z_{1}(s)\right)-F\left(s, Z_{2}(s)\right)\right)\right| d s+$

$+\left|\int_{0}^{t}\right| \Psi(t) X(t) P_{0} X^{-1}(s) \Psi^{-1}(s) \| \Psi(s)\left(F\left(s, Z_{1}(s)\right)-F\left(s, Z_{2}(s)\right)\right)|d s|+$

$+\int_{t}^{\infty}\left|\Psi(t) X(t) P_{+} X^{-1}(s) \Psi^{-1}(s) \| \Psi(s)\left(F\left(s, Z_{1}(s)\right)-F\left(s, Z_{2}(s)\right)\right)\right| d s \leq$

$\leq \gamma \int_{-\infty}^{t}\left|\Psi(t) X(t) P_{-} X^{-1}(s) \Psi^{-1}(s)\right|\left|\Psi(s)\left(Z_{1}(s)-Z_{2}(s)\right)\right| d s+$

$+\gamma\left|\int_{0}^{t}\right| \Psi(t) X(t) P_{0} X^{-1}(s) \Psi^{-1}(s)|| \Psi(s)\left(Z_{1}(s)-Z_{2}(s)\right)|d s|+$

$+\gamma \int_{t}^{\infty}\left|\Psi(t) X(t) P_{+} X^{-1}(s) \Psi^{-1}(s)\right|\left|\Psi(s)\left(Z_{1}(s)-Z_{2}(s)\right)\right| d s \leq$

$\leq \gamma K \sup _{t \in R}\left|\Psi(s)\left(Z_{1}(s)-Z_{2}(s)\right)\right|=\gamma K\left|Z_{1}-Z_{2}\right|_{\Psi}$. 
It follows that

$$
\left|T Z_{1}-T Z_{2}\right|_{\Psi} \leq \gamma K\left|Z_{1}-Z_{2}\right|_{\Psi} .
$$

Therefore, $\mathrm{T}$ is a contraction operator on $S_{\rho}$. Hence, by Banach fixed point theorem, $\mathrm{T}$ has a unique fixed point $Z \in S_{\rho}$. From $Z=T Z$, it follows that $\mathrm{Z}$ is continuous differentiable on $\mathrm{R}$ and then, for $t \in R$,

$$
\begin{aligned}
& Z^{\prime}(t)=(T Z)^{\prime}(t)= \\
& =\int_{-\infty}^{t} X^{\prime}(t) P_{-} X^{-1}(s)(C(s)+F(s, Z(s))) d s+ \\
& +X(t) P_{-} X^{-1}(t)(C(t)+F(t, Z(t)))+ \\
& +\int_{0}^{t} X^{\prime}(t) P_{0} X^{-1}(s)(C(s)+F(s, Z(s))) d s+ \\
& +X(t) P_{0} X^{-1}(t)(C(t)+F(s, Z(t)))- \\
& -\int_{t}^{\infty} X^{\prime}(t) P_{+} X^{-1}(s)(C(s)+F(s, Z(s))) d s+ \\
& +X(t) P_{+} X^{-1}(t)(C(t)+F(t, Z(t)))= \\
& =A(t)(T Z)(t)+X(t)\left(P_{-}+P_{0}+P_{+}\right) X^{-1}(t)(C(t)+F(t, Z(t)))= \\
& =A(t) Z(t)+C(t)+F(t, Z(t)) .
\end{aligned}
$$

Thus, $\mathrm{Z}(\mathrm{t})$ is a solution of equation (1.2).

In conclusion, the equation (1.2) has a unique $\Psi$ - bounded solution $Z(t)$ on $R$ for which $|\Psi(t) Z(t)| \leq \rho$, for all $t \in R$.

Remark 3.1. Theorem generalizes the Theorem 4 ([5], Ch. 5, s. 2) and Theorem 2.1, [18] from systems of differential equations to matrix differential equations and extents them for case $P_{0} \neq 0$.

The next simple example is an illustration of Theorem.

Example 3.1. Consider the equation (1.2) with

$$
A(t)=\operatorname{diag}[-2 t, 1,-1], C(t)=\operatorname{diag}\left[\frac{\alpha}{1+t^{2}}, \alpha e^{-t} \sin t, \alpha e^{t} \cos t\right]
$$

and

$$
F(t, Z)=\operatorname{diag}\left[\ln \left(1+a\left|z_{11}\right|\right), \sin a z_{22}, \operatorname{arctg} a z_{33}\right],
$$

where $Z=\left(z_{i j}\right) \in \mathbb{M}_{3 \times 3}$ and $a, \alpha$ are real constants such that

$$
0<a<\left[1+\int_{0}^{1} e^{s^{2}} d s\right]^{-1} .
$$


Then, $\mathrm{X}(\mathrm{t})=\operatorname{diag}\left[e^{t^{2}}, e^{t}, e^{-t}\right], t \in R$, is a fundamental matrix for (2.3).

Consider $\Psi(\mathrm{t})=\operatorname{diag}\left[1, e^{t}, e^{-t}\right], t \in R$.

There exist supplementary projections

$$
P_{-}=\operatorname{diag}[0,0,1], P_{0}=\operatorname{diag}[1,0,0], \text { and } P_{+}=\operatorname{diag}[0,1,0]
$$

such that:

- $\Psi(t) X(t) P_{-} X^{-1}(s) \Psi^{-1}(s)=\operatorname{diag}\left[0,0, e^{-2(t-s)}\right]$ and then

$$
\int_{-\infty}^{t}\left|\Psi(t) X(t) P_{-} X^{-1}(s) \Psi^{-1}(s)\right| d s \leq \int_{-\infty}^{t} e^{-2(t-s)} d s=\frac{1}{2} ;
$$

- $\Psi(t) X(t) P_{+} X^{-1}(s) \Psi^{-1}(s)=\operatorname{diag}\left[0, e^{2(t-s)}, 0\right]$ and then

$$
\int_{t}^{\infty}\left|\Psi(t) X(t) P_{+} X^{-1}(s) \Psi^{-1}(s)\right| d s \leq \int_{t}^{\infty} e^{2(t-s)} d s=\frac{1}{2} ;
$$

- $\Psi(t) X(t) P_{0} X^{-1}(s) \Psi^{-1}(s)=\operatorname{diag}\left[e^{-t^{2}+s^{2}}, 0,0\right]$ and then

$$
\begin{aligned}
& \left|\int_{0}^{t}\right| \Psi(t) X(t) P_{0} X^{-1}(s) \Psi^{-1}(s)|d s|=\left|\int_{0}^{t} e^{-t^{2}+s^{2}} d s\right|= \\
= & \int_{0}^{|t|} e^{-t^{2}+s^{2}} d s \leq 1+\int_{0}^{1} e^{s^{2}} d s .
\end{aligned}
$$

and then, the condition (3.1) is satisfied with $K=1+\int_{0}^{1} e^{s^{2}} d s$.

After that, for $t \in R$ and for $Z^{\prime}, Z^{\prime \prime} \in \mathbb{M}_{3 \times 3}$, we have

$$
\begin{aligned}
& \left|\Psi(t)\left(F\left(t, Z^{\prime}\right)-F\left(t, Z^{\prime \prime}\right)\right)\right| \leq \\
& \leq \max \left\{a\left|z_{11}^{\prime}-z_{11}^{\prime \prime}\right|, a e^{t}\left|z_{22}^{\prime}-z_{22}^{\prime \prime}\right|, a e^{-t}\left|z_{33}^{\prime}-z_{33}^{\prime \prime}\right|\right\}= \\
& =a \cdot \max \left\{\left|z_{11}^{\prime}-z_{11}^{\prime \prime}\right|, e^{t}\left|z_{22}^{\prime}-z_{22}^{\prime \prime}\right|, e^{-t}\left|z_{33}^{\prime}-z_{33}^{\prime \prime}\right|\right\}= \\
& =a \cdot\left|\Psi(t)\left(Z^{\prime}-Z^{\prime \prime}\right)\right|
\end{aligned}
$$

and then, the condition 2) of Theorem is satisfied.

At least, for the matrix $C(t)$ we have that

$$
|C|=\sup _{t \in R}|\Psi(t) C(t)|=|\alpha| .
$$

From Theorem, it follows that for $\rho \geq \frac{|\alpha| K}{1-a K}$, the equqtion (1.2) has a unique $\Psi$ - bounded solution $Z(t)$ for which $|\Psi(t) Z(t)| \leq \rho$, for all $t \in R$. 
Theorem 3.2. Suppose that:

1). There exist supplementary projections $P_{-}, P_{+} \in \mathbb{M}_{d \times d}$ and a positive constants $K_{1}, K_{2}, \alpha$ and $\beta$ such that the fundamental matrix $X(t)$ for (2.3) satisfies the conditions

$$
\begin{aligned}
& \left|\Psi(t) X(t) P_{-} X^{-1}(s) \Psi^{-1}(s)\right| \leq K_{1} e^{-\alpha(t-s)}, \text { for } s \leq t \\
& \left|\Psi(t) X(t) P_{+} X^{-1}(s) \Psi^{-1}(s)\right| \leq K_{2} e^{-\beta(s-t)}, \text { for } t \leq s
\end{aligned}
$$

2). The continuous function $F: R \times \mathbb{M}_{d \times d} \rightarrow \mathbb{M}_{d \times d}$ satisfies the condition

$$
|\Psi(t) F(t, Z)| \leq \gamma|\Psi(t) Z|,
$$

for $t \in R, Z \in \mathbb{M}_{d \times d}$ with $|\Psi(t) Z| \leq \rho$ for $t \in R$ ( $\rho>0$ is given), where $\gamma$ is a positive constant such that $\gamma\left(\frac{K_{1}}{\alpha}+\frac{K_{2}}{\beta}\right)<1$;

3). The continuous function $C: R \rightarrow \mathbb{M}_{d \times d}$ is $\Psi$ - bounded on $R$ such that

$$
|C|_{\Psi}=\sup _{t \in R}|\Psi(t) C(t)| \leq \frac{\rho\left[1-\gamma\left(\frac{K_{1}}{\alpha}+\frac{K_{2}}{\beta}\right)\right]}{\frac{K_{1}}{\alpha}+\frac{K_{2}}{\beta}} .
$$

Then, the equation (1.2) has at least one $\Psi$ - bounded solution $Z(t)$ on $R$ for which $|\Psi(t) Z(t)| \leq \rho$, for all $t \in R$.

Proof. We prove this treorem by means of Schauder-Tychonoff fixed point theorem.

For this, let $C_{\Psi}$ denote the set of all matrix functions $Z(t)$ which are continuous and $\Psi$-bounded on $\mathrm{R}$, and $S_{\rho}$ be the subset formed by those functions $Z(t)$ such that $|Z|_{\Psi}=\sup _{t \in R}|\Psi(t) Z(t)| \leq \rho$.

For $Z \in C_{\Psi}$, define the operator $\mathrm{T}$ by

$$
\begin{aligned}
& (T Z)(t)=\int_{-\infty}^{t} X(t) P_{-} X^{-1}(s)(C(s)+F(s, Z(s))) d s- \\
& -\int_{t}^{\infty} X(t) P_{+} X^{-1}(s)(C(s)+F(s, Z(s))) d s
\end{aligned}
$$

This operator have the following two properties:

i). $\mathrm{T}$ is continuous, in the sense that if $Z_{n} \in S_{\rho}(n=1,2, \ldots)$ and $Z_{n} \rightarrow Z$ uniformly on every compact subinterval $\mathrm{J}$ of $\mathrm{R}$, then $T Z_{n} \rightarrow T Z$ uniformly on every compact subinterval $\mathrm{J}$ of $\mathrm{R}$.

Indeed, let $Z_{n} \in S_{\rho}(n=1,2, \ldots)$ and $Z_{n} \rightarrow Z$ uniformly on every compact subinterval $J=[p, q]$ of $\mathrm{R}$. For an arbitrary small $\varepsilon>0$, choose $\tau>0$ so large that

$$
\tau>\max \left\{-\frac{1}{\alpha} \ln \frac{\alpha \varepsilon}{8 \rho \gamma K_{1}},-\frac{1}{\beta} \ln \frac{\beta \varepsilon}{8 \rho \gamma K_{2}}\right\} .
$$


Since $F(t, Z)$ is uniformly continuous for $t \in[p-\tau, q+\tau]$ and $|\Psi(t) Z| \leq \rho$, it follows that the sequence $U_{n}(t)=\Psi(t)\left(F\left(t, Z_{n}(t)\right)-F(t, Z(t))\right)$ tends to zero uniformly on $[p-\tau, q+\tau]$. Thus, there exists $n_{0} \in \mathbb{N}$ such that $\left|U_{n}(t)\right|<\frac{\varepsilon}{4 \tau \max \left\{K_{1}, K_{2}\right\}}$, for $n \geq n_{0}$ and $t \in[p-\tau, q+\tau]$.

For $t \in J$ and $n \geq n_{0}$, consider

$$
\begin{aligned}
& \left|\Psi(t)\left(\left(T Z_{n}\right)(t)-(T Z)(t)\right)\right|= \\
& =\mid\left[\int_{-\infty}^{t} \Psi(t) X(t) P_{-} X^{-1}(s) \Psi^{-1}(s) \Psi(s)\left(C(s)+F\left(s, Z_{n}(s)\right)\right) d s-\right. \\
& \left.-\int_{t}^{\infty} \Psi(t) X(t) P_{+} X^{-1}(s) \Psi^{-1}(s) \Psi(s)\left(C(s)+F\left(s, Z_{n}(s)\right)\right) d s\right]- \\
& -\left[\int_{-\infty}^{t} \Psi(t) X(t) P_{-} X^{-1}(s) \Psi^{-1}(s) \Psi(s)(C(s)+F(s, Z(s))) d s-\right. \\
& \left.-\int_{t}^{\infty} \Psi(t) X(t) P_{+} X^{-1}(s) \Psi^{-1}(s) \Psi(s)(C(s)+F(s, Z(s))) d s\right] \mid= \\
& =\mid \int_{-\infty}^{t} \Psi(t) X(t) P_{-} X^{-1}(s) \Psi^{-1}(s) \Psi(s)\left(F\left(s, Z_{n}(s)\right)-F(s, Z(s))\right) d s- \\
& -\int_{t}^{\infty} \Psi(t) X(t) P_{+} X^{-1}(s) \Psi^{-1}(s) \Psi(s)\left(F\left(s, Z_{n}(s)\right)-F(s, Z(s))\right) d s \mid \leq \\
& \leq \int_{-\infty}^{t}\left|\Psi(t) X(t) P_{-} X^{-1}(s) \Psi^{-1}(s)\right|\left|\Psi(s)\left(F\left(s, Z_{n}(s)\right)-F(s, Z(s))\right)\right| d s+ \\
& +\int_{t}^{\infty}\left|\Psi(t) X(t) P_{+} X^{-1}(s) \Psi^{-1}(s)\right|\left|\Psi(s)\left(F\left(s, Z_{n}(s)\right)-F(s, Z(s))\right)\right| d s \leq \\
& \leq K_{1} \int_{-\infty}^{t} e^{-\alpha(t-s)}\left|U_{n}(s)\right| d s+K_{2} \int_{t}^{\infty} e^{-\beta(s-t)}\left|U_{n}(s)\right| d s= \\
& =K_{1} \int_{-\infty}^{t-\tau} e^{-\alpha(t-s)}\left|U_{n}(s)\right| d s+K_{1} \int_{t-\tau}^{t} e^{-\alpha(t-s)}\left|U_{n}(s)\right| d s+ \\
& +K_{2} \int_{t+\tau}^{\infty} e^{-\beta(s-t)}\left|U_{n}(s)\right| d s+K_{2} \int_{t}^{t+\tau} e^{-\beta(s-t)}\left|U_{n}(s)\right| d s \leq \\
& \leq 2 \rho \gamma\left(K_{1} \int_{-\infty}^{t-\tau} e^{-\alpha(t-s)} d s+K_{2} \int_{t+\tau}^{\infty} e^{-\beta(s-t)} d s\right)+\max \left\{K_{1}, K_{2}\right\} \int_{t-\tau}^{t+\tau}\left|U_{n}(s)\right| d s< \\
& <2 \rho \gamma\left(K_{1} \cdot \frac{e^{-\alpha \tau}}{\alpha}+K_{2} \cdot \frac{e^{-\beta \tau}}{\beta}\right)+\max \left\{K_{1}, K_{2}\right\} \cdot \frac{\varepsilon}{4 \tau \max \left\{K_{1}, K_{2}\right\}} \cdot 2 \tau<\varepsilon
\end{aligned}
$$

This shows that $T Z_{n} \rightarrow T Z$ uniformly on every compact subinterval of $\mathrm{R}$. Thus, $\mathrm{T}$ is continuous.

ii). the functions in the image set $T S_{\rho}$ are equicontinuous and bounded at every point of $J$.

Indeed, from $Z \in S_{\rho}$, we have 


$$
\begin{aligned}
& |\Psi(t)(T Z)(t)|= \\
& =\mid \int_{-\infty}^{t} \Psi(t) X(t) P_{-} X^{-1}(s) \Psi^{-1}(s) \Psi(s)(C(s)+F(s, Z(s))) d s- \\
& -\int_{t}^{\infty} \Psi(t) X(t) P_{+} X^{-1}(s) \Psi^{-1}(s) \Psi(s)(C(s)+F(s, Z(s))) d s \mid \leq \\
& \leq \int_{-\infty}^{t}\left|\Psi(t) X(t) P_{-} X^{-1}(s) \Psi^{-1}(s) \| \Psi(s)(C(s)+F(s, Z(s)))\right| d s+ \\
& +\int_{t}^{\infty}\left|\Psi(t) X(t) P_{+} X^{-1}(s) \Psi^{-1}(s)\right||\Psi(s)(C(s)+F(s, Z(s)))| d s \leq \\
& \leq K_{1} \int_{-\infty}^{t} e^{-\alpha(t-s)}|\Psi(s)(C(s)+F(s, Z(s)))| d s+ \\
& +K_{2} \int_{t}^{\infty} e^{-\beta(s-t)}|\Psi(s)(C(s)+F(s, Z(s)))| d s \leq \\
& \leq K_{1} \int_{-\infty}^{t} e^{-\alpha(t-s)}(|\Psi(s) C(s)|+\gamma|\Psi(s) Z(s)|) d s+ \\
& +K_{2} \int_{t}^{\infty} e^{-\beta(s-t)}(|\Psi(s) C(s)|+\gamma|\Psi(s) Z(s)|) d s \leq \\
& \leq\left(\frac{\rho\left[1-\gamma\left(\frac{K_{1}}{\alpha}+\frac{K_{2}}{\beta}\right)\right]}{\frac{K_{1}}{\alpha}+\frac{K_{2}}{\beta}}+\gamma \rho\right)\left(\frac{K_{1}}{\alpha}+\frac{K_{2}}{\beta}\right)=\rho .
\end{aligned}
$$

Hence, the functions in the image set $T S_{\rho}$ are uniformly bounded at every point of $J$.

On the other hand, we have

$$
\begin{aligned}
& (T Z)^{\prime}(t)= \\
& =\int_{-\infty}^{t} X^{\prime}(t) P_{-} X^{-1}(s)(C(s)+F(s, Z(s))) d s+ \\
& +X(t) P_{-} X^{-1}(t)(C(t)+F(t, Z(t)))+ \\
& -\int_{t}^{\infty} X^{\prime}(t) P_{+} X^{-1}(s)(C(s)+F(s, Z(s))) d s+ \\
& +X(t) P_{+} X^{-1}(t)(C(t)+F(t, Z(t)))= \\
& =A(t)(T Z)(t)+X(t)\left(P_{-}+P_{+}\right) X^{-1}(t)(C(t)+F(t, Z(t)))= \\
& =A(t)(T Z)(t)+C(t)+F(t, Z(t)),
\end{aligned}
$$

which shows that $(T Z)(t)$ is a solution of equation $W^{\prime}=A(t) W+C(t)+$ $F(t, Z(t))$. 
It follows that the derivatives $(T Z)^{\prime}(t)$ are uniformly bounded on any compact subinterval $\mathrm{J}$ of $\mathrm{R}$. Thus, the functions in $T S_{\rho}$ are echicontinuous on any compact subinterval $\mathrm{J}$ of $\mathrm{R}$.

From i) and ii), all the conditions of the Schauder-Tychonoff theorem are satisfied. Hence, the operator $\mathrm{T}$ has at least one fixed point $\mathrm{Z}(\mathrm{t})$ in $S_{\rho}$. But the fixed point of $\mathrm{T}$ is just the solution of the integral equation

$$
Z=T Z
$$

in $S_{\rho}$, i.e. , of the matrix differential equation (1.2), with the required properties.

Remark 3.2. In a particular case, our result reduces to Theorem 2.2 obtained in [18].

Indeed, if

$$
F(t, Z)=\left(\begin{array}{cccc}
f_{1}(t, z) & f_{1}(t, z) & \cdots & f_{1}(t, z) \\
f_{2}(t, z) & f_{2}(t, z) & \cdots & f_{2}(t, z) \\
\vdots & \vdots & \vdots & \vdots \\
f_{d}(t, z) & f_{d}(t, z) & \cdots & f_{d}(t, z)
\end{array}\right), C(t)=\left(\begin{array}{llll}
c_{1}(t) & c_{1}(t) & \cdots & c_{1}(t) \\
c_{2}(t) & c_{2}(t) & \cdots & c_{2}(t) \\
\vdots & \vdots & \vdots & \vdots \\
c_{d}(t) & c_{d}(t) & \cdots & c_{d}(t)
\end{array}\right)
$$

it is easy to see that the solutions of the equation (1.2) is

$$
Z(t)=\left(\begin{array}{cccc}
z_{1}(t) & z_{1}(t) & \cdots & z_{1}(t) \\
z_{2}(t) & z_{2}(t) & \cdots & z_{2}(t) \\
\vdots & \vdots & \vdots & \vdots \\
z_{d}(t) & z_{d}(t) & \cdots & z_{d}(t)
\end{array}\right)
$$

where $z(t)=\left(z_{1}(t), z_{2}(t), \cdots, z_{d}(t)\right)^{T}$ is the solution of the equation (1.3) with

$c(t)=\left(c_{1}(t), c_{2}(t), \cdots, c_{d}(t)\right)^{T}$ and $f(t, z)=\left(f_{1}(t, z), f_{1}(t, z), \cdots, f_{1}(t, z)\right)^{T}$.

In this case, the solution $z(t)$ is $\Psi$-bounded on $\mathrm{R}$ iff the corresponding solution $Z(t)$ is $\Psi$-bounded on $\mathrm{R}$.

Thus, the Theorem generalizes the result from [18], from systems of differential equations to matrix differential equations. 


\section{$4 \Psi$ - bounded solutions for the Lyapunov matrix dif- ferential equation (1)}

The purpose of this section is to provide sufficient conditions for the existence and uniqueness and existence of at least one $\Psi$ - bounded solution on $\mathbb{R}$ for the Lyapunov matrix differential equation (1.1).

Theorem 4.1. Suppose that:

1). There exist supplementary projections $P_{-}, P_{0}, P_{+} \in \mathbb{M}_{d \times d}$ and a positive constant $K$ such that the fundamental matrices $X(t)$ and $Y(t)$ for (2.3) and (2.4) respectively satisfy the condition

$$
\begin{aligned}
& \int_{-\infty}^{t}\left|\left(Y^{T}(t)\left(Y^{T}\right)^{-1}(s)\right) \otimes\left(\Psi(t) X(t) P_{-} X^{-1}(s) \Psi^{-1}(s)\right)\right| d s+ \\
& +\left|\int_{0}^{t}\right|\left(Y^{T}(t)\left(Y^{T}\right)^{-1}(s)\right) \otimes\left(\Psi(t) X(t) P_{0} X^{-1}(s) \Psi^{-1}(s)\right)|d s|+ \\
& +\int_{t}^{\infty}\left|\left(Y^{T}(t)\left(Y^{T}\right)^{-1}(s)\right) \otimes\left(\Psi(t) X(t) P_{+} X^{-1}(s) \Psi^{-1}(s)\right)\right| d s \leq K,
\end{aligned}
$$

for all $t \geq 0$;

2). The continuous function $F: R \times \mathbb{M}_{d \times d} \rightarrow \mathbb{M}_{d \times d}$ satisfies the Lypschitz condition

$$
\left|\Psi(t)\left(F\left(t, Z_{1}\right)-F\left(t, Z_{2}\right)\right)\right| \leq \frac{\gamma}{d}\left|\Psi(t)\left(Z_{1}-Z_{2}\right)\right|,
$$

for $t \in R, Z_{1}, Z_{2} \in \mathbb{M}_{d \times d}$ with $\left|\Psi(t) Z_{1}\right| \leq \rho,\left|\Psi(t) Z_{2}\right| \leq \rho$ for $t \in R, \quad(\rho>0$ is given), where $\gamma$ is a positive constant such that $\gamma K<1$;

3). The continuous matrix function $C: R \rightarrow \mathbb{M}_{d \times d}$ is $\Psi$ - bounded on $R$ such that

$$
|C|_{\Psi}=\sup _{t \in R}|\Psi(t) C(t)| \leq \frac{\rho(1-\gamma K)}{K} .
$$

Then, the Lyapunov matrix differential equation (1.1) has a unique $\Psi-$ bounded solution $Z(t)$ on $R$ for which $|\Psi(t) Z(t)| \leq \rho d$, for all $t \in R$.

Proof. From Lemma 2.4, one know that $\mathrm{Z}(\mathrm{t})$ is a solution of (1.1) iff the vector function $z(t)=\operatorname{Vec}(Z(t))$ is a solution of the corresponding Kronecker product system associated with (1.1), i.e. of the differential system (2.1).

From Lemma (2.7), one know that $U(t)=Y^{T}(t) \otimes X(t)$ is a fundamental matrix for the differential system (2.5).

Now, the hypotheses of the Theorem ensure the hypotheses of Theorem 3.1 (variant for systems) for the system (2.1). Indeed: 
i). Since

$$
\begin{aligned}
& \left(Y^{T}(t)\left(Y^{T}\right)^{-1}(s)\right) \otimes\left(\Psi(t) X(t) P_{-} X^{-1}(s) \Psi^{-1}(s)\right)= \\
& =(I \otimes \Psi(t)) \cdot\left(Y^{T}(t) \otimes X(t)\right) \cdot\left(I \otimes P_{-}\right) \cdot\left(\left(Y^{T}\right)^{-1}(s) \otimes X^{-1}(s)\right) \cdot\left(I \otimes \Psi^{-1}(s)\right)
\end{aligned}
$$

(see Lemma 2.1) and similarly for $P_{0}$ and $P_{+}$, the hypothesis 1 ) of Theorem 3.1 is satisfied;

ii). Since

$$
\begin{aligned}
& \left\|(I \otimes \Psi(t)) \cdot\left(f\left(t, z_{1}\right)-f\left(t, z_{2}\right)\right)\right\|_{R^{d^{2}}}= \\
& =\left\|(I \otimes \Psi(t)) \cdot \operatorname{Vec}\left(F\left(t, Z_{1}\right)-F\left(t, Z_{2}\right)\right)\right\|_{R^{d^{2}}} \leq \\
& \leq\left|\Psi(t)\left(F\left(t, Z_{1}\right)-F\left(t, Z_{2}\right)\right)\right| \leq \frac{\gamma}{d}\left|\Psi(t)\left(Z_{1}-Z_{2}\right)\right| \leq \\
& \leq \gamma\left\|(I \otimes \Psi(t)) \cdot \operatorname{Vec}\left(Z_{1}-Z_{2}\right)\right\|_{R^{d^{2}}}= \\
& =\gamma\left\|(I \otimes \Psi(t)) \cdot\left(z_{1}-z_{2}\right)\right\|_{R^{d^{2}}},
\end{aligned}
$$

for all $z_{1}, z_{2}$ with $\left\|(I \otimes \Psi(t)) \cdot z_{i}\right\|_{R^{d^{2}}}=\left\|(I \otimes \Psi(t)) \cdot \operatorname{Vec}\left(Z_{i}\right)\right\|_{R^{d^{2}}} \leq 1$ $\Psi(t) Z_{i} \mid \leq \rho$,

(see Lemmas 2.1 and 2.5) and $\gamma K<1$, the hypothesis 2) of Theorem 3.1 is satisfied;

iii). Since

$$
\begin{aligned}
& \|c\|_{R^{d^{2}}}=|C|=\sup _{t \in R}|(I \otimes \Psi(t)) \operatorname{Vec}(C(t))| \leq \\
& \leq \sup _{t \in R}|\Psi(t) C(t)| \leq \frac{\rho(1-\gamma K)}{K},
\end{aligned}
$$

the hypothesis 3) of Theorem 3.1 is satisfied.

At this stage we appeal to Theorem 3.1 to deduce that the system (2.1) has a unique $I \otimes \Psi(t)$ - bounded solution $z(t)$ on R for which $\|(I \otimes \Psi(t))$. $z(t) \|_{R^{d^{2}}} \leq \rho$.

From Lemma 2.4 again, the matrix function $Z(t)=V e c^{-1}(z(t))$ is unique solution of (1.1) on $\mathrm{R}$ such that (see Lemma 2.5) $|\Psi(t) Z(t)| \leq \rho d$, for all $t \in R$.

Remark 4.1. The Theorem extends the Theorem 2.1, [18] and Theorem 3.1 above to Lyapunov matrix differential equation (1.1).

Theorem 4.2. Suppose that:

1). There exist supplementary projections $P_{-}, P_{+} \in \mathbb{M}_{d \times d}$ and a positive 
constants $K_{1}, K_{2}, \alpha$ and $\beta$ such that the fundamental matrices $X(t)$ and $Y(t)$ for (2.3) and (2.4) respectively satisfy the condition

$$
\begin{aligned}
& \left|\left(Y^{T}(t)\left(Y^{T}\right)^{-1}(s)\right) \otimes\left(\Psi(t) X(t) P_{-} X^{-1}(s) \Psi^{-1}(s)\right)\right| \leq K_{1} e^{-\alpha(t-s)}, \text { for } s \leq t \\
& \left|\left(Y^{T}(t)\left(Y^{T}\right)^{-1}(s)\right) \otimes\left(\Psi(t) X(t) P_{+} X^{-1}(s) \Psi^{-1}(s)\right)\right| \leq K_{2} e^{-\beta(s-t)}, \text { for } t \leq s
\end{aligned}
$$

2). The continuous function $F: R \times \mathbb{M}_{d \times d} \rightarrow \mathbb{M}_{d \times d}$ satisfies the condition

$$
|\Psi(t) F(t, Z)| \leq \frac{\gamma}{d}|\Psi(t) Z|
$$

for $t \in R, Z \in \mathbb{M}_{d \times d}$ with $|\Psi(t) Z| \leq \rho$ for $t \in R$ ( $\rho>0$ is given), where $\gamma$ is a positive constant such that $\gamma\left(\frac{K_{1}}{\alpha}+\frac{K_{2}}{\beta}\right)<1$;

3). The continuous matrix function $C: R \rightarrow \mathbb{M}_{d \times d}$ is $\Psi$ - bounded on $R$ such that

$$
|C|_{\Psi}=\sup _{t \in R}|\Psi(t) C(t)| \leq \frac{\rho\left[1-\gamma\left(\frac{K_{1}}{\alpha}+\frac{K_{2}}{\beta}\right)\right]}{\frac{K_{1}}{\alpha}+\frac{K_{2}}{\beta}} .
$$

Then, the Lyapunov matrix differential equation (1.1) has at least one $\Psi-$ bounded solution $Z(t)$ on $R$ for which $|\Psi(t) Z(t)| \leq \rho d$.

Proof. From Lemma 2.4, one know that $\mathrm{Z}(\mathrm{t})$ is a solution of (1.1) iff the vector function $z(t)=\operatorname{Vec}(Z(t))$ is a solution of the corresponding Kronecker product system associated with (1.1), i.e. of the differential system (2.1).

From Lemma (2.7), one know that $U(t)=Y^{T}(t) \otimes X(t)$ is a fundamental matrix for the differential system (2.5).

Now, the hypotheses of the Theorem ensure the hypotheses of Theorem 3.2 (variant for systems) for the system (2.1). Indeed:

i). Since

$$
\begin{aligned}
& \left(Y^{T}(t)\left(Y^{T}\right)^{-1}(s)\right) \otimes\left(\Psi(t) X(t) P_{-} X^{-1}(s) \Psi^{-1}(s)\right)= \\
& =(I \otimes \Psi(t)) \cdot\left(Y^{T}(t) \otimes X(t)\right) \cdot\left(I \otimes P_{-}\right) \cdot\left(\left(Y^{T}\right)^{-1}(s) \otimes X^{-1}(s)\right) \cdot\left(I \otimes \Psi^{-1}(s)\right)
\end{aligned}
$$

(see Lemma 2.1) and similarly for $P_{+}$, the hypothesis 1 ) of Theorem 3.2 is satisfied;

ii). Since

$$
\begin{aligned}
& \|(I \otimes \Psi(t)) \cdot f(t, z)\|_{R^{d^{2}}}=\|(I \otimes \Psi(t)) \cdot \operatorname{Vec} F(t, Z)\|_{R^{d^{2}}} \leq \\
& \leq|\Psi(t) F(t, Z)| \leq \frac{\gamma}{d}|\Psi(t) Z| \leq \gamma\|(I \otimes \Psi(t)) \cdot \operatorname{Vec}(Z)\|_{R^{d^{2}}}= \\
& =\gamma\|(I \otimes \Psi(t)) \cdot z\|_{R^{d^{2}}},
\end{aligned}
$$


for $t \in R$ and $z \in R^{d^{2}}$, (see Lemmas 2.1 and 2.5) and $\gamma\left(\frac{K_{1}}{\alpha}+\frac{K_{2}}{\beta}\right)<1$, the hypothesis 2) of Theorem 3.2 is satisfied;

iii). Since

$$
\begin{aligned}
& \|c\|_{R^{d^{2}}}=\|\operatorname{Vec}(C(t))\|_{R^{d^{2}}}=\sup _{t \in R}|(I \otimes \Psi(t)) \operatorname{Vec}(C(t))| \leq \\
& \leq \sup _{t \in R}|\Psi(t) C(t)| \leq \frac{\rho\left(1-\gamma\left(\frac{K_{1}}{\alpha}+\frac{K_{2}}{\beta}\right)\right)}{\frac{K_{1}}{\alpha}+\frac{K_{2}}{\beta}},
\end{aligned}
$$

the hypothesis 3) of Theorem 3.2 is satisfied.

At this stage we appeal to Theorem 3.2 to deduce that the system (2.1) has at least one $I \otimes \Psi(t)$ - bounded solution $z(t)$ on $\mathrm{R}$ for which $\|(I \otimes \Psi(t))$. $z(t) \|_{R^{d^{2}}} \leq \rho$.

From Lemma 2.4 again, the matrix function $Z(t)=V e c^{-1}(z(t))$ is a solution of (1.1) on R such that (see Lemma 2.5), $|\Psi(t) Z(t)| \leq \rho d$, for all $t \in R$.

Remark 4.2. The Theorem extends the Theorem 2.2 [18] and Theorem 3.2 above to Lyapunov matrix differential equation (1.1).

The next simple example is an illustration of Theorem.

Example 4.1. Consider the nonlinear Lyapunov matrix differential equation (1.1) with

$$
\begin{gathered}
A(t)=\left(\begin{array}{cc}
2 & 0 \\
0 & -2
\end{array}\right), B(t)=\left(\begin{array}{ll}
1 & 0 \\
0 & 0
\end{array}\right) \\
C(t)=\left(\begin{array}{cc}
0 & a e^{4 t} \cos |t| \\
a e^{-3 t} \sin t^{2} & a e^{-3|t|}
\end{array}\right)
\end{gathered}
$$

and

$$
F(t, Z)=m\left(\begin{array}{cc}
\sin z_{1} \sin t & z_{2} \cos t \\
z_{3} \sin z_{4} & \frac{2}{\pi} z_{4} \operatorname{arctg} t
\end{array}\right),
$$

where $t \in R, Z=\left(\begin{array}{ll}z_{1} & z_{2} \\ z_{3} & z_{4}\end{array}\right) \in \mathbb{M}_{2 \times 2}$ and a, m are real constants, $0<|m|<$ $\frac{1}{4}$.

Then,

$$
X(t)=\left(\begin{array}{cc}
e^{2 t} & 0 \\
0 & e^{-2 t}
\end{array}\right) \text { and } Y(t)=\left(\begin{array}{cc}
e^{t} & 0 \\
0 & 1
\end{array}\right)
$$

are fundamental matrices for (2.3) and (2.4) respectively.

Consider

$$
\Psi(t)=\left(\begin{array}{cc}
e^{-4 t} & 0 \\
0 & e^{3 t}
\end{array}\right), t \in R
$$


There exist supplementary projections

$$
P_{-}=\left(\begin{array}{cc}
1 & 0 \\
0 & 0
\end{array}\right) \text { and } P_{+}=\left(\begin{array}{cc}
0 & 0 \\
0 & 1
\end{array}\right)
$$

such that

and

$$
\left|\left(Y^{T}(t)\left(Y^{T}\right)^{-1}(s)\right) \otimes\left(\Psi(t) X(t) P_{-} X^{-1}(s) \Psi^{-1}(s)\right)\right|=e^{-\alpha(t-s)}, \text { for } s \leq t
$$

$$
\left|\left(Y^{T}(t)\left(Y^{T}\right)^{-1}(s)\right) \otimes\left(\Psi(t) X(t) P_{+} X^{-1}(s) \Psi^{-1}(s)\right)\right|=e^{-(s-t)}, \text { for } t \leq s .
$$

Thus, the condition 1) of Theorem is satisfied with $K_{1}=K_{2}=1$ and $\alpha=\beta=1$.

After that, for $t \in R, Z=\left(\begin{array}{cc}z_{1} & z_{2} \\ z_{3} & z_{4}\end{array}\right) \in \mathbb{M}_{2 \times 2}$, we have

$|\Psi(t) F(t, Z)|=\left|m\left(\begin{array}{ll}e^{-4 t} \sin z_{1} \sin t & e^{-4 t} z_{2} \cos t \\ e^{3 t} z_{3} \sin z_{4} & \frac{2}{\pi} e^{3 t} z_{4} \operatorname{arctg} t\end{array}\right)\right| \leq|m||\Psi(t) Z|$.

Thus, the condition 2) of Theorem is satisfied with $\gamma=2|m|$ and $d=2$.

At least, for the matrix $C(t)$ we have that

$$
|C|=\sup _{t \in R}|\Psi(t) C(t)|=\sup _{t \in R}\left|\left(\begin{array}{cc}
0 & a \cos |t| \\
a \sin t^{2} & a e^{-3|t|+3 t}
\end{array}\right)\right|=2|a| .
$$

Now, from Theorem 4.2, it follows that for all $\rho \geq \frac{8|a|}{1-4|m|}$, the equation (1.1) has at least one solution $Z(t)$ for which $|\Psi(t) Z(t)| \leq \rho$, for all $t \in R$.

\section{References}

[1] R. Bellman, Introduction to Matrix Analysis, McGraw-Hill Book Company, Inc. New York, 1960 (translated in Romanian).

[2] P. N. Boi, Existence of $\Psi$ - bounded solutions on R for nonhomogeneous linear differential equations, Electron. J. Diff. Eqns. 2007 (2007), 52:110 .

[3] P. N. Boi, On the $\Psi$-dichotomy for homogeneous linear differential equations, Electronic Journal of Differential Equations 2006 (2006), 40: $1-12$. 
[4] A. Constantin, Asymptotic properties of solutions of differential equations, Analele Universităţii din Timişoara, Seria Ştiinte Matematice $\mathbf{3 0}$ (2-3) (1992), 183-225.

[5] W. A. Coppel, Stability and Asymptotic Behavior of Differential Equations, D. C. Heath and Company, Boston, 1965.

[6] A. Diamandescu, On the $\Psi$ - boundedness of the solutions of a nonlinear Lyapunov matrix differential equation, Applied Sciences 19 (2017), $31-40$.

[7] A. Diamandescu, On the $\Psi$ - boundedness of the solutions of linear nonhomogeneous Lyapunov matrix differential equations, Differential Geometry - Dynamical Systems 19 (2017), 35-44.

[8] A. Diamandescu, A note on the existence of $\Psi$ - bounded solutions for a system of differential equations on R, Electronic Journal of Differential Equations 2008 (2008), 128: 1-11.

[9] A. Diamandescu, $\Psi$ - bounded solutions for linear differential systems with Lebesgue $\Psi$ - integrable functions on $\mathrm{R}$ as right-hand sides, Electronic Journal of Differential Equations 2009 (2009), 5: 1-12.

[10] A. Diamandescu, $\Psi$ - bounded solutions for a Lyapunov matrix differential equation, Electronic Journal of Qualitative Theory Differential Equations 17 (2009), 1-11.

[11] A. Diamandescu, Existence of $\Psi$ - bounded solutions for nonhomogeneous Lyapunov matrix differential equations on R, Electronic Journal of Qualitative Theory Differential Equations 42 (2010), 1-9.

[12] A. Diamandescu, On the $\Psi$ - boundedness of the solutions of a linear system of ordinary differential equations, Analele Ştiinţifice ale Universității "Al. I. Cuza" Iaşi, XLVIII, s. I, Matematică, (2) (2002), 269-286.

[13] T. G. Hallam, On asymptotic equivalence of the bounded solutions of two systems of differential equations, Mich. Math. Journal 16 (1969), 353-363.

[14] T. Hara, T. Yoneyama, T. Itoh, Asymptotic Stability Criteria for Nonlinear Volterra Integro-Differential Equations, Funkcialaj Ekvacioj 33 (1990), 39-57. 
[15] J. R. Magnus, H. Neudecker, Matrix Differential Calculus with Applications in Statistics and Econometrics, John Wiley \& Sons Ltd, Chichester, 1999.

[16] M. S. N. Murty, G. Suresh Kumar, On $\Psi$ - boundedness and $\Psi$ - stability of matrix Lyapunov systems, J. Appl. Math. Comput. 26 (2008), $67-84$

[17] M. S. N. Murty, G. Suresh Kumar, On $\Psi$ - bounded solutions for non-homogeneous matrix Lyapunov systems on R, Electronic Journal of Qualitative Theory Differential Equations 62 (2009), 1-12.

[18] Ch. Vasavi, T. Srinivasa Rao, G. Suresh Kumar, On $\Psi$ - bounded solutions for semi-linear differential equations on R, International Journal of Advances in Engineering, Science and Technology 3, No. 1 (2013), $22-25$

Aurel Diamandescu

Department of Applied Mathematics

University of Craiova

13 A.I. Cuza Street

200585, Craiova

Romania

E-mail: diamandescu.aurel@ucv.ro 